\title{
Applications of Atomic Force Microscopy in Biological Research
}

\author{
Joseph M. Wallace ${ }^{1,2}$
}

1. Department of Biomedical Engineering, Indiana University Purdue University of Indianapolis, Indianapolis, USA

2. Department of Orthopaedic Surgery, Indiana University School of Medicine, Indianapolis, USA

Most musculoskeletal tissues are hierarchical, meaning that structural elements can be defined which, themselves, have structure on smaller length scales. Hierarchy plays an important role in determining overall mechanical properties and hierarchical structures typically contain less material to achieve a desired strength. A real-word example of structural hierarchy is the Eiffel Tower, which reaches over 300 meters tall with a square base of 100 meters per side. Each leg is constructed as a lattice-work of beams composed of girders with cross-sectional dimensions of $10 \mathrm{~s}$ to $100 \mathrm{~s}$ of centimeters. These sections are made of a material with an atomic structure of ions organized in an Angstrom-scale lattice structure. In a similar manner, bone has structural elements which span 9-10 orders of magnitude in length (Figure 1). At its smallest hierarchical level, bone is a two-phase composite composed of a relatively soft and ductile collagen phase which is impregnated with and surrounded by a stiffer reinforcing mineral phase. The nanoscale size of these constituents inhibits the roles of each from being completely understood. Adding cellular and metabolic aspects further complicates the issue.

The Bone Biology and Mechanics Lab at IUPUI is focused on understanding how biological and environmental factors influence bone assembly and organization with a goal of developing rational approaches for disease diagnosis and treatment. We use multiscale approaches to investigate composition, architecture, mechanical integrity, and fracture resistance at discrete length scales throughout the hierarchy of bone in the context of disease, following external mechanical stimulation or during pharmaceutical treatment. One of the unique features of our program, and a common thread that passes through all of our research, is an intense focus on collagen and the roles it plays in bone health. Studying collagen requires the use of technologies which let us peer into the nanoscale world.

Scanning probe microscopy has been used for over 30 years to assess nanoscale features in a variety of engineering and biological materials. A form of STM known as atomic force microscopy (AFM) was introduced in 1986. AFM works by raster-scanning a sharpened probe which is mounted to a flexible cantilever across a sample's surface. As the probe scans in the plane of the sample, the cantilever deflects to accommodate the changing surface topography. This up and down movement generates a high-resolution image. AFM can also take advantage of the fact that its cantilever is a sensitive force transducer and can be used to extract nanoscale mechanical information. As new and easy to use AFM systems have become readily available, the technique has made its way into the mainstream. However, many take a "black box" approach to AFM which can lead to a host of issues. The goal of this special topic session will be to discuss some of the strengths and limitations of using AFM in biological research. It will cover technical hurdles associated with imaging and indentation work. All topics will be discussed with an eye towards potential pitfalls which could jeopardize data quality and interpretation.

When using AFM to image or indent biological samples, the choice of which features to measure, how to measure them, proper site selection and sampling strategies are all important factors to consider. In general, proper sample hydration is also an important but often difficult issue to contend with. A 


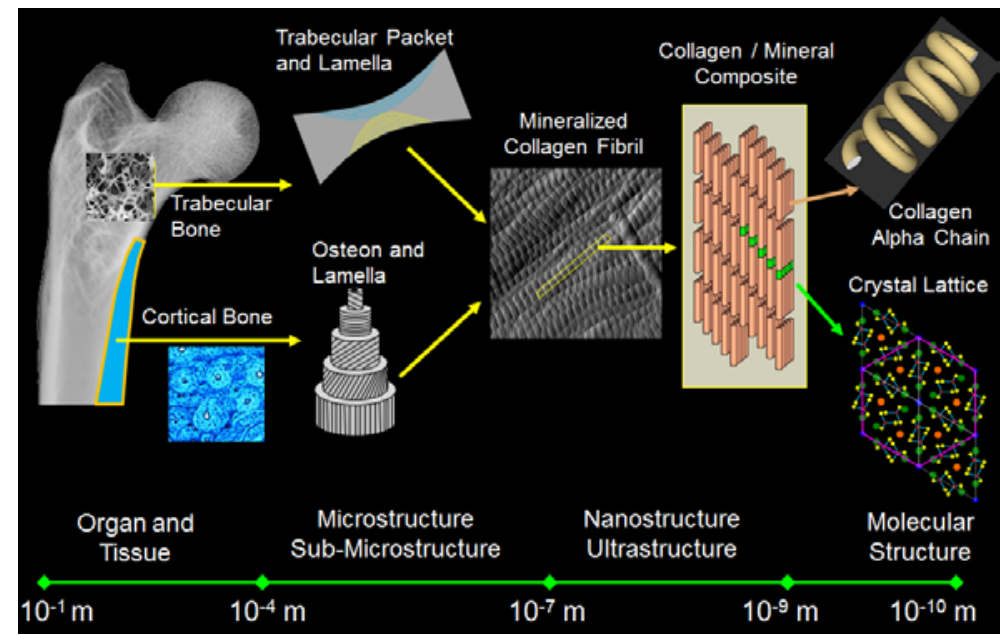

Figure 1: Schematic representation of the hierarchical structure of bone. Note the nanoscale size of bone's fundamental constituents (collagen and mineral).

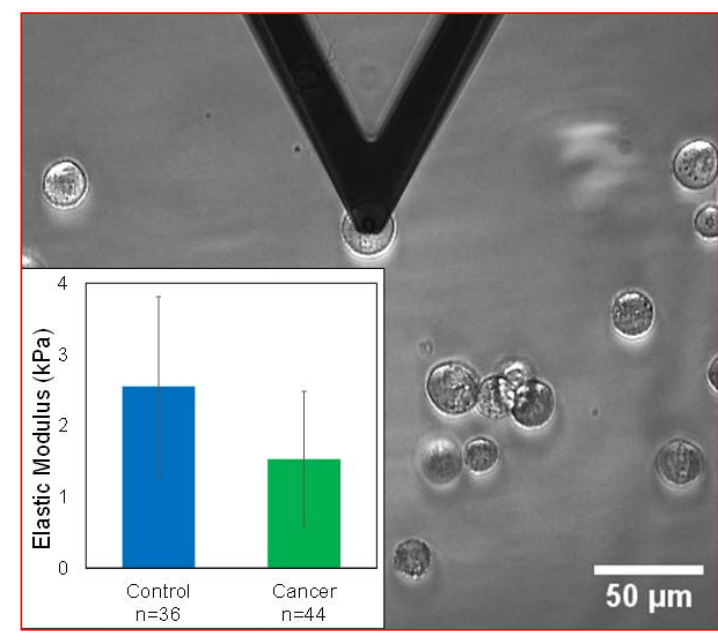

Figure 2: A soft cantilever equipped with a $5 \mu \mathrm{m}$ diameter borosilicate bead was used to indent normal and cancer cells. Elastic modulus was significantly decreased in the cancer cells $(\mathrm{p}<0.001)$.

significant, and often overlooked consideration, is proper calibration. Since a strength of AFM is the ability to accurately measure nanoscale features, calibration using scan and feature sizes comparable to features of interest is highly recommended for all applications. For indentation work, these same issues need to be considered, in addition to a host of other factors. Proper cantilever and probe selection for the intended application are critical (e.g. a stiff cantilever coupled with a sharp probe may poke rather than deform the sample). An accurate spring constant must be obtained for each cantilever, as should the radius of curvature or tip half angle. Finally, to extract useful properties, a proper force range and mechanical fitting model must be selected. Taking these factors into consideration, AFM can be used for a range of biological applications. As an example, using large probes and soft cantilevers, cells were indented to discriminate between normal and cancel cells (Figure 2) [1]. When equipped with sharper and stiffer probes, intact fascicles from mouse tail tendons were imaged to reveal the rich collagen structure with its prominent banding (Figure 3) [2]. These samples were subsequently indented revealing that drying collagen increases its elastic modulus by 2 orders of magnitude.

Without understanding the assumptions and limitations of the technique, it is easy to generate less than ideal data using AFM. However, with a little knowledge and careful application, AFM can provide true nanoscale morphological and mechanical information which cannot be obtained by other means.

\section{References}

1. TruongVo T et al., J of Micromechanics and Microengineering (2017) In Press.

2. Kemp A et al., J of Structural Biology. 180 (2012), 428-38.

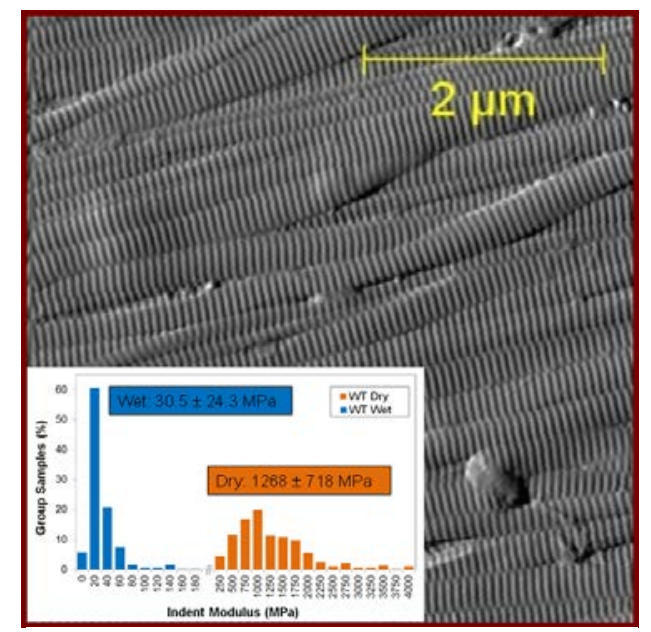

Figure 3: AFM was used to image and indent tail tendon fascicles and demonstrated that when dried, elastic modulus was increased by 2 orders of magnitude $(\mathrm{p}<0.001)$. 